\title{
Osteocondroma: diagnóstico radiológico, complicaciones y variantes
}

\author{
Dres. Marco Cañete P, Elena Fontoira M, Begoña Gutiérrez San José, Slavina Mancheva M.
}

Servicio de Radiología Músculo-esquelética. Fundación Jiménez Díaz. Madrid, España.

\section{Osteochondroma: Diagnostic imaging, complications and variants}

\begin{abstract}
Understanding and recognising the spectrum of appearances of osteochondroma is important because it represents the most frequent pseudotumoral bone lesion. There are pathognomonic radiological features that are evident with the currently available imaging methods. The recognition of these features and their potential complications and variants, enables a correct diagnosis to be made, the identification of possible complications and is a guide for the therapeutic decisions of non-conclusive cases.
\end{abstract}

Keywords: Bone tumors, Dysplasia, Exostosis, Osteochondroma.

Resumen: Conocer el espectro de apariencias del osteocondroma es importante, ya que representa lesión pseudotumoral más frecuente del hueso y posee unas características radiológicas patognomónicas evidenciables con los distintos métodos de imagen disponibles actualmente. El reconocimiento de estas características radiológicas, de sus posibles complicaciones y variantes permite establecer el diagnóstico correcto, identificar las posibles complicaciones y guiar el manejo terapéutico de los casos no concluyentes. Palabras clave: Displasia, Exostosis, Neurológicas, Osteocondroma, Tumores óseos.

Cañete M, et al. Osteocondroma: diagnóstico radiológico, complicaciones y variantes. Rev Chil 2013; $19(2): 73-81$. Correspondencia: Marco A. Cañete Prette / MACañete@fjd.es

Trabajo recibido 28 de noviembre de 2012, aceptado para publicación el 2 de junio del 2013.

\section{Introducción}

El osteocondroma representa la lesión pseudotumoral más frecuente del hueso. La característica radiológica patognomónica de este tumor es la continuidad cortical y medular de las lesiones con el hueso del que surgen ${ }^{(1)}$. Las lesiones pueden ser solitarias o múltiples, formando esta última parte del síndrome de exostosis hereditaria múltiple ${ }^{(2)}$. Estas lesiones también se pueden presentar con complicaciones como deformidades óseas, fracturas ${ }^{(1,3)}$, compromiso neurológico o vascular ${ }^{(1,4,5)}$, formación de bursa y más raramente transformación maligna ${ }^{(6,7)}$. Las variantes más comunes incluyen la exostosis subungueal, la displasia epifisaria hemimielica (enfermedad de Trevor $^{(1,8)}$, la proliferación osteocondromatosa paraostal (lesión de Nora) ${ }^{(9)}$ y la periostitis reactiva florida. En general, el diagnóstico puede ser realizado con una radiografía, de acuerdo a las características del tumor, aunque los otros métodos de imagen como la ecografía, el TAC y en especial la RM pueden ser de utilidad ante casos dudosos, o ante la aparición de lesiones sintomáticas o en lugares poco comunes ${ }^{(1)}$. El objetivo de este trabajo es ilustrar las diversas presentaciones del osteocondroma, así como sus complicaciones y variantes.

\section{Fisiopatología y epidemiología}

Se considera a los osteocondromas lesiones del desarrollo más que verdaderas neoplasias ${ }^{(1)}$. La lesión, de acuerdo a la OMS, está definida como una exostosis osteocartilaginosa con continuidad cortical y medular, respectivamente ${ }^{(10)}$. Se piensa que estas lesiones resultan de la separación de un fragmento del cartílago de crecimiento epifisario, que se va herniando a través del hueso normal que rodea el platillo de crecimiento(1).

En el caso de los osteocondromas múltiples se ha descrito su asociación con mutaciones de los genes EXT 1 y EXT 2, que intervienen en la biosíntesis de proteoglicanos heparán sulfato, involucrados en el crecimiento epifisiario, y con la radiación, que podría producir desdiferenciación del tejido cartilaginoso 
del crecimiento, entre otros ${ }^{(1)}$. En general, los osteocondromas se presentan mayormente en niños o adolescentes, sin predilección por sexo, aunque algunos autores consideran una mayor frecuencia en el sexo masculino ${ }^{(10,11)}$.

\section{Osteocondroma solitario}

La gran mayoría de los osteocondromas son lesiones solitarias ${ }^{(1)}$. Los sitios más comunes de aparición son los huesos largos del miembro inferior, mayormente en el extremo distal del fémur y proximal de la tibia ${ }^{(12)}$ (Figura 1) y en el miembro superior, el húmero ${ }^{(1)}$. Lugares más raros de aparición descritos en la literatura abarcan los huesos de las manos y pies, la escápula, pelvis, la columna vertebral|(1,13-15) (Figuras 2 a,b y 3 a,b), las costillas y lugares todavía más raros como la articulación temporomandibular ${ }^{(16,17)}$ o tejidos blandos como la grasa de Hoffa alrededor de la rodilla ${ }^{(18)}$ (Figuras $\left.4 a, b\right)$, aunque hay que tener en cuenta que cualquier hueso que se forme desde osificación encondral podría desarrollarlo(1). La mayoría son lesiones asintomáticas ${ }^{(18)}$ y descubiertas incidentalmente, y si se presentan con síntomas, el más común de ellos es el desarrollo de un bultoma de lento crecimiento(1).

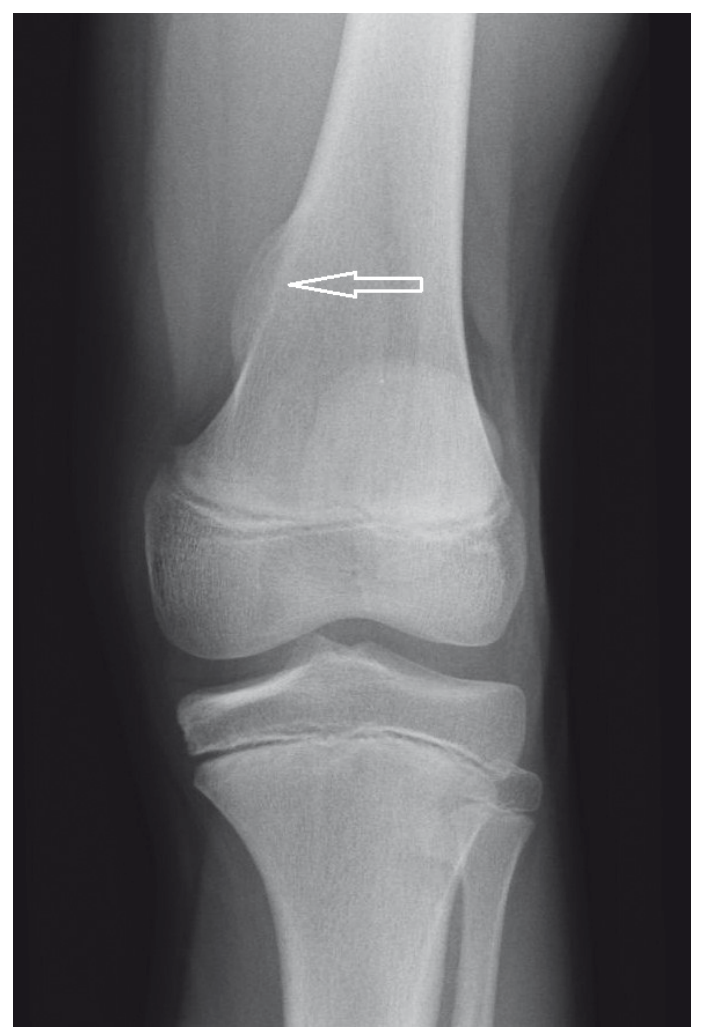

Figura 1. $R X A P$ de rodilla: se observa una lesión ósea cortical exofítica en unión metafiso-diafisaria distal medial del fémur, con continuidad cortico medular (flecha).

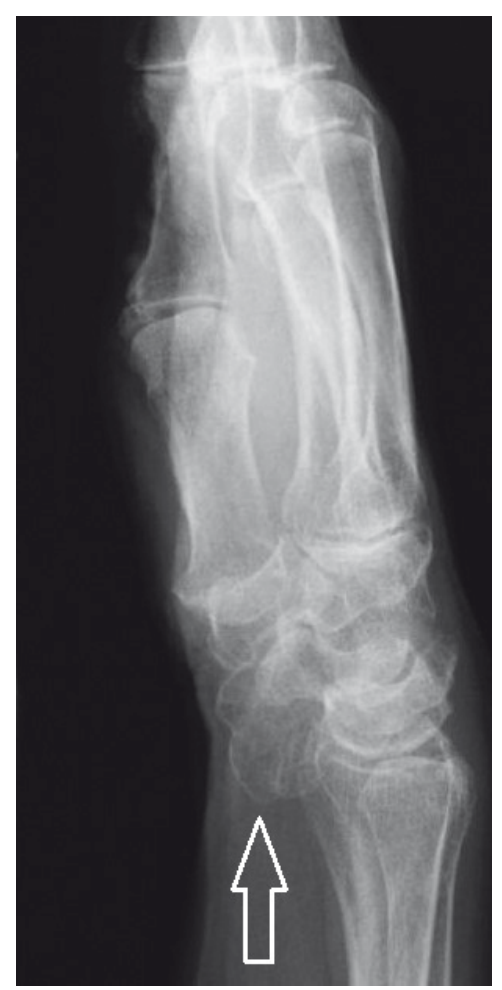

Figura 2a. $R X$ lateral de muñeca: Osteocondroma del pisiforme: lesión ósea exofítica lobulada de aspecto benigno dependiente del hueso pisiforme (flecha).

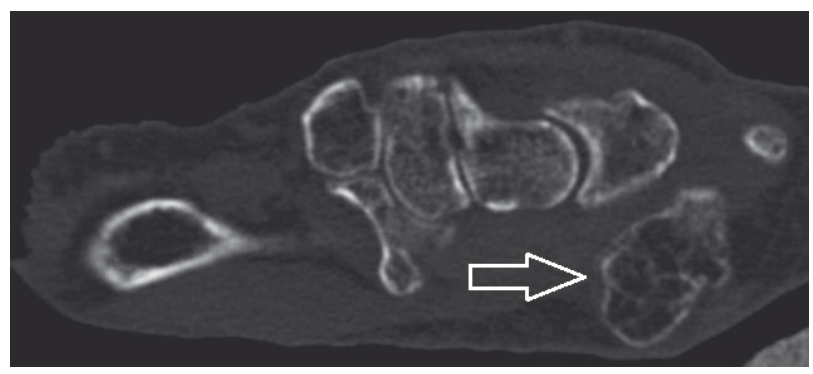

Figura 2b. TC axial de muñeca con reconstrucción ósea: Osteocondroma del pisiforme: lesión ósea cortical exofítica y lobulada dependiente del hueso pisiforme de aspecto benigno (flecha).

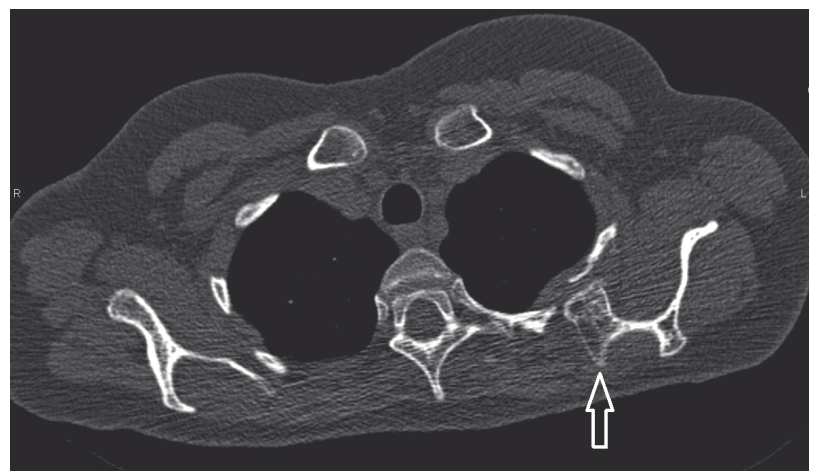

Figura 3a. TC axial de tórax con reconstrucción ósea: Osteocondroma escapular: lesión ósea cortical con aspecto benigno dependiente del borde medial de la escápula izquierda (flecha). 


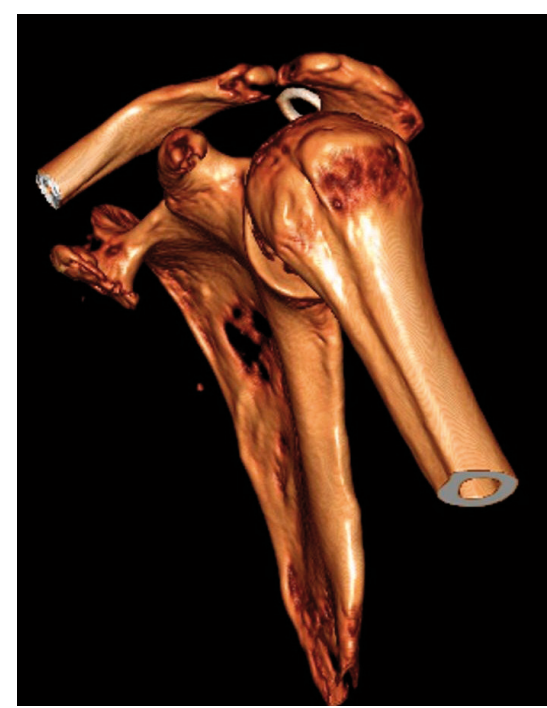

Figura 3 b. TC escápula reconstrucción 3D: Osteocondroma escapular: lesión ósea exofítica sésil dependiente de borde medial de la escápula.

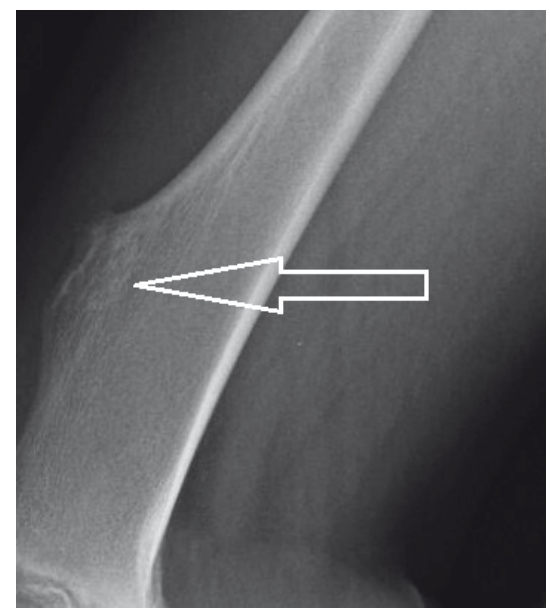

Figura 4a. $R X$ de rodilla lateral: Osteocondroma femoral: lesión ósea cortical exofític a dependiente de unión metafisodiafisaria de fémur distal (flecha).

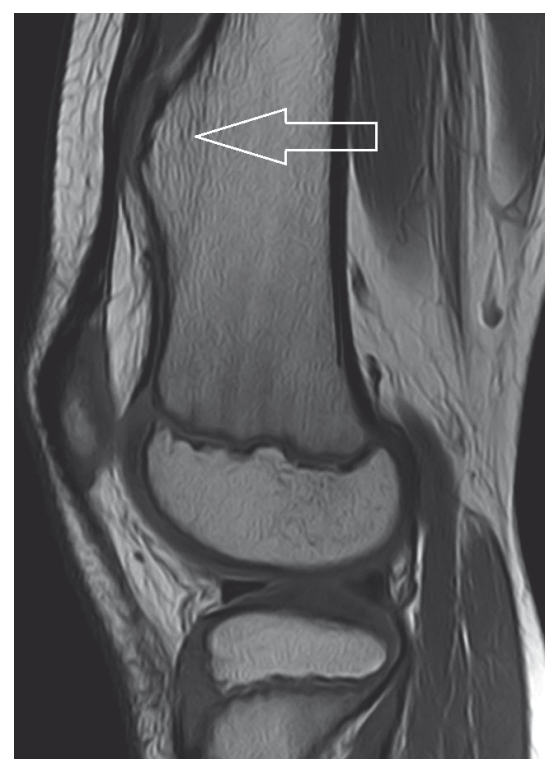

Figura 4 b. $R M$ de rodilla de rech a, secuencia Sagital T1: Osteocondroma sésil de fémur distal: lesión exofítica en diáfisis femoral distal con continuidad cortico medular (flecha)

Las características radiológicas son bastante típicas, visualizándose una lesión exofítica con continuidad cortical y medular con el hueso adyacente del que surge ${ }^{(19)}$. Respecto a su base de implantación, ésta puede ser ancha o estrecha, lo que representa los dos tipos de osteocondroma solitario, el sésil y el pedunculado (Figuras 5 y 6), siendo la radiografía muchas veces suficiente para el diagnóstico ${ }^{(1)}$. La localización más frecuente en huesos largos suelen ser las metáfisis y los lugares de inserción tendino$\mathrm{sa}^{(10)}$. Sin embargo, en huesos planos el diagnóstico puede ser más difícil. La capa de cartílago hialino puede ser visualizada en la radiografía como áreas de calcificaciones en punto y coma, pero en casos con ausencia de mineralización pueden ser necesarios otros métodos de imagen. La ecografía permite la visualización de la capa cartilaginosa, que se visualiza como un área hipoecoica sobre la corteza del hueso estudiado, pero demuestra más utilidad para el estudio de complicaciones como aneurismas, trombosis o bursitis ${ }^{(10,20)}$. Respecto al TC, éste permite una excelente visualización de la continuidad corticomedular de la lesión, y puede ser todavía más útil en áreas de anatomía compleja, como los huesos de la columna, el hombro o la pelvis ${ }^{(10)}$. Sin embargo, algunos autores consideran que este método de imagen puede no ser adecuado en la medición del grosor de la capa cartilaginosa, criterio importante para la diferenciación con el condrosarcoma ${ }^{(1)}$. Por último, la RM permite una mejor visualización que la radiografía de la continuidad corticomedular de lesiones en áreas complejas ${ }^{(1)}$. La continuidad cortical se visualiza como una línea fina hipointensa en todas las secuencias de pulso, y la médula mantiene señal de médula amarilla (Figuras 7 a,b). Además, se considera a la RM como el mejor método para la visualización de las estructuras circundantes a la lesión, su efecto sobre éstas (compromiso vascular y nervioso), datos de complicación (pseudoaneurismas, edema) y la capa de cartílago no mineralizada, que demuestra una señal alta en T1 y alta en T2, debido a su contenido en agua, permitiendo estas características la medición adecuada de su grosor ${ }^{(1)}$ (Figura 8). Hay que tener en cuenta que la presencia de edema es un signo de complicación y podría indicar la presencia de un condrosarcoma ${ }^{(10)}$.

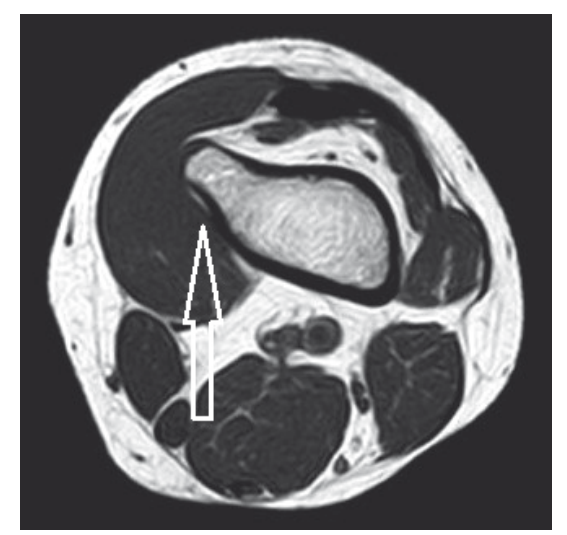

Figura 5. RM Axial de muslo, secuencia Axial T1: Osteocondroma sésil de fémur distal: Lesión ósea exofítica dependiente de diáfisis femoral (flecha), con continuidad cortical y medular 


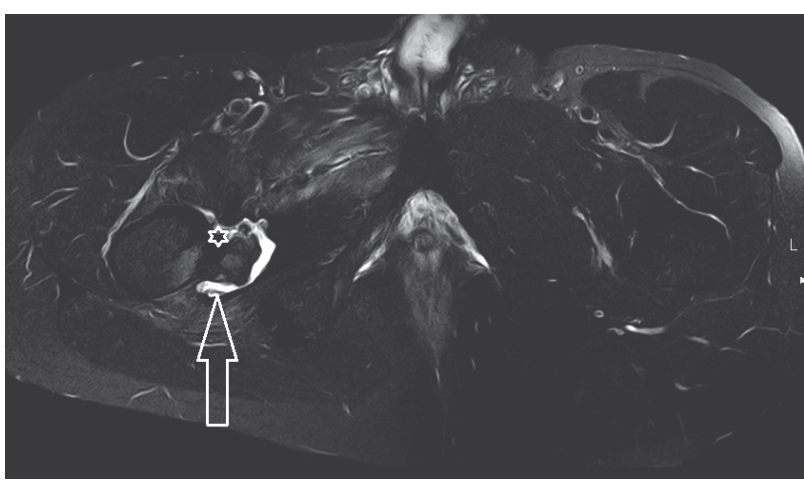

Figura 6. RM Axial de cadera, secuencia T2 Spir: Osteocondroma pedunculado: tumoración ósea (flecha) pedunculada (asterisco) irregular, que depende del borde posterior del cuello del fémur derecho, con continuidad cortico medular

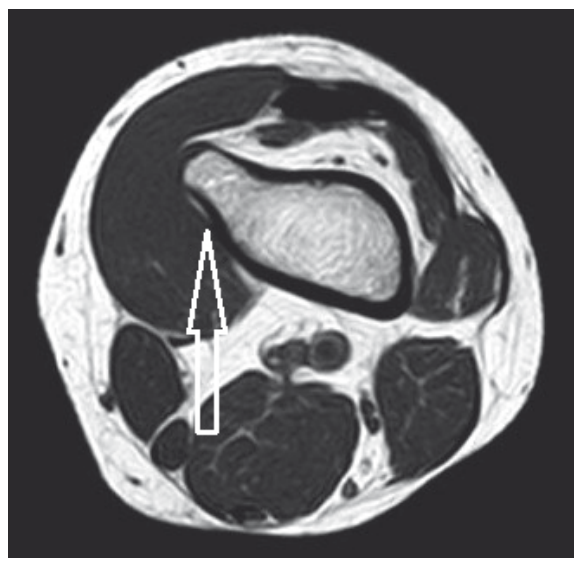

Figura 7a. RM Axial de Muslo, secuencia T1: Osteocondroma sésil de fémur: lesión ósea cortical exofítica (flecha) dependiente de diáfisis femoral distal con continuidad cortico medular.

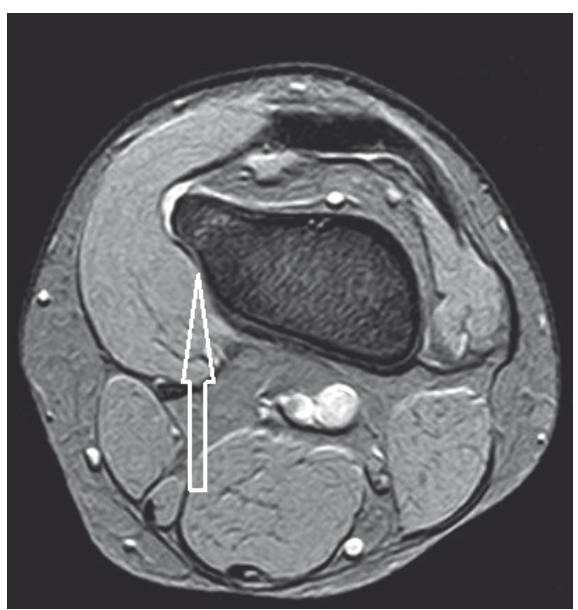

Figura 7b. RM Axial de Muslo, secuencia T2: Osteocondroma sésil de fémur: lesión ósea cortical exofítica (flecha) dependiente de diáfisis femoral distal con continuidad cortico medular.

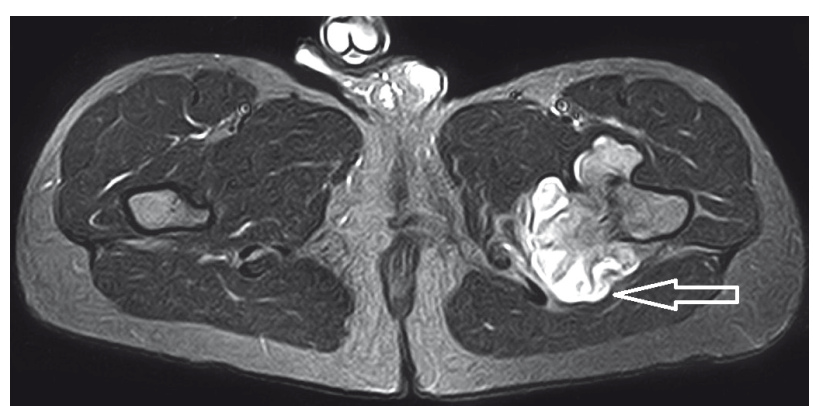

Figura 8.RM Axial de cadera, secuencia T2 Stir: Osteocondroma sésil de fémur: lesión ósea dependiente de fémur izquierdo, visualizándose capa de cartílago hialino hiperintensa (flecha).

\section{Exostosis hereditaria múltiple (EHM)}

Es un síndrome caracterizado por el desarrollo de múltiples osteocondromas y muestra un patrón genético autosómico dominante ${ }^{(1)}$. Algunos autores consideran diagnóstica la presencia radiológica de al menos dos osteocondromas en regiones yuxtaepifisarias de huesos largos. Se considera que la aparición de osteocondromas solitarios es seis veces más frecuente que en este síndrome. La clínica y las complicaciones son las mismas que las lesiones únicas, aunque los pacientes con esta afección pueden presentar una variedad de deformidades ortopédicas, siendo la más frecuente entre éstas el acortamiento del antebrazo ${ }^{(2,21)}$. La presencia de complicaciones es mayor en este síndrome, ya que existe mayor número de lesiones ${ }^{(1)}$. Se cree que la base genética de este síndrome está relacionada a mutaciones de los genes EXT 1 y $2^{(2)}$. La complicación más temida es la malignización de los osteocondromas a condrosarcomas, como veremos más adelante, aunque cabe decir que se piensa que esta transformación es más frecuente en osteocondromas de pacientes que presentan este síndrome ${ }^{(10)}$. En el diagnóstico diferencial hay que tener en cuenta a la enfermedad de Trevor, ya que tanto ésta como el osteocondroma se diagnostican a edades tempranas y su desarrollo se relaciona con el cierre de la placa de crecimiento. Sin embargo, las lesiones en la enfermedad de Trevor, afectan a los miembros inferiores unilateralmente y se limitan al lado medial o lateral de este (hemimélica). Por último, no se ha reportado malignización de las lesiones en esta última ${ }^{(2)}$.

\section{Complicaciones del osteocondroma}

Entre las complicaciones más frecuentes se encuentran la presencia de deformidades óseas, fracturas, compromiso vascular y neurológico, la formación de bursa y la malignización ${ }^{(22,23)}$.

Deformidad ósea: se considera la forma de presentación más común y suele ser más frecuente en pacientes con EHM. Suele afectar mayormente a las rodillas, caderas y el tobillo, en orden de frecuencia ${ }^{(1)}$. 
Debido al efecto de masa, en algunos casos, se puede observar erosión sobre el hueso adyacente y en el caso de contacto con vísceras, éstas también pueden encontrarse afectadas como, por ejemplo, en el caso de osteocondromas de los arcos costales, que pueden producir derrame pleural y/o hemotórax ${ }^{(1,24)}$.

Fractura: Otra complicación a tener en cuenta y, consecuentemente, produce dolor, lo que puede ser una forma de presentación ${ }^{(10)}$, siendo más frecuente en las lesiones pedunculadas a nivel de su base ${ }^{(1)}$. Teniendo en cuenta el efecto de masa que pueden producir estas lesiones, siendo exofiticas, no es infrecuente que se produzcan complicaciones como síndromes de compresión vascular o nerviosa, y, de hecho, éstos se pueden presentar de diversas formas (Figura 9 a-c).

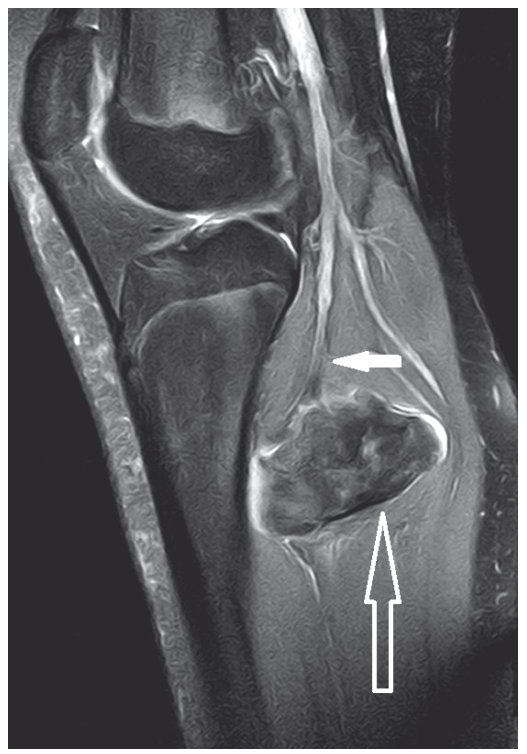

Figura 9a. RM sagital de rodilla, secuencia DP Spir: Osteocondroma del peroné: lesión ósea exofítica dependiente del peroné (flecha hueca), con efecto de masa con desplazamiento de paquete vasculonervioso femoral posterior (flecha).

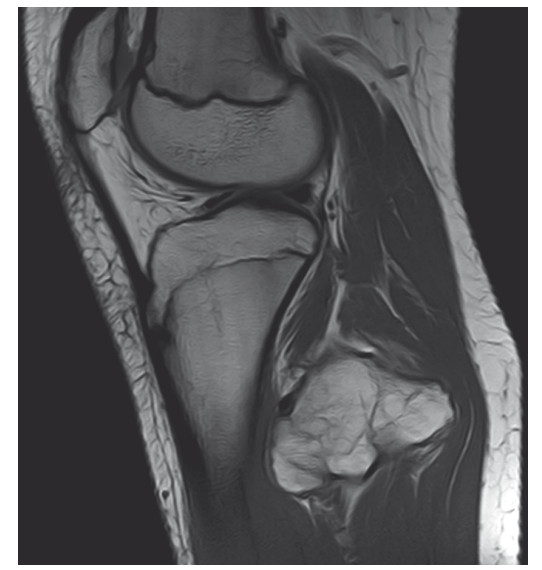

Figura 9b. RM sagital de rodilla, secuencia T1: Osteocondroma del peroné: se observa el efecto de masa a nivel del compartimiento muscular posterior de la pantorrilla.

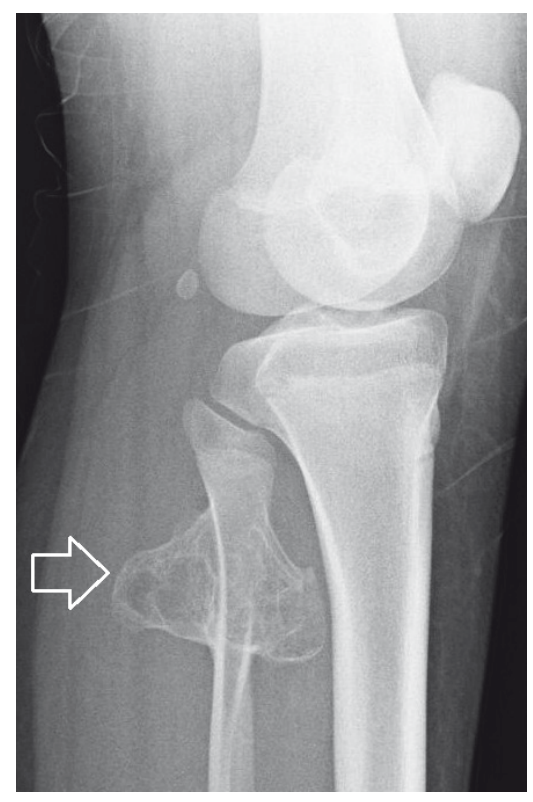

Figura 9c. Rx lateral de rodilla: Osteocondroma del peroné: lesión ósea exofítica cortical dependiente de unión metafisodiafisaria posterior del peroné, con continuidad cortico medular (flecha).

Síndromes vasculares: se puede producir desplazamiento de vasos (Figura 10), estenosis, oclusión vascular y formación de pseudoaneurismas, siendo estos últimos más frecuente a nivel de la rodilla, involucrando generalmente a la arteria poplítea ${ }^{(1,25)}$ o presentarse como una trombosis arterial ${ }^{(26)}$. Por último, en el caso de lesiones costales, se ha descrito su presentación como síndrome del estrecho torácico debido a la oclusión de vasos adyacentes ${ }^{(27)}$.

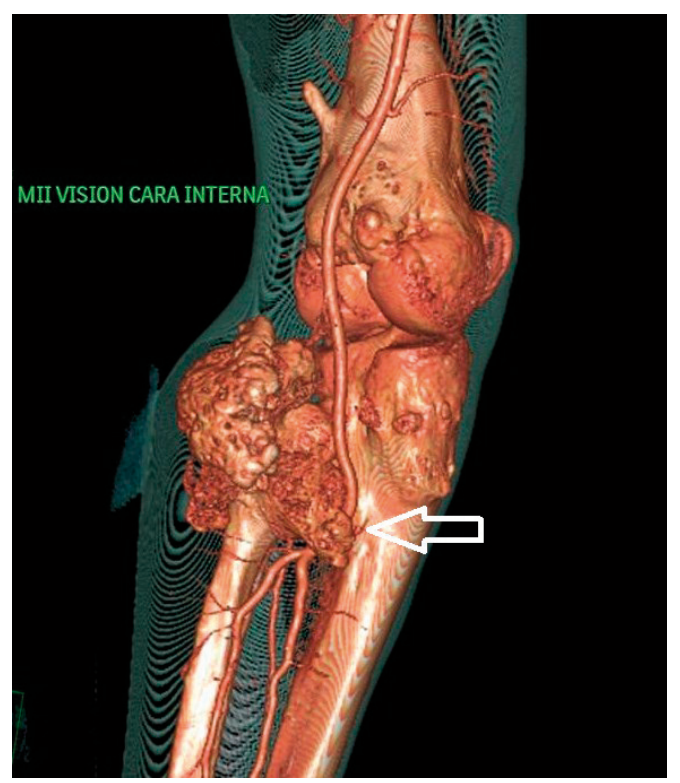

Figura 10. AngioTC de MMII, reconstrucción 3D: lesión ósea tibioperonea (flecha) que produce evidente desplazamiento del tronco tibioperoneo ipsilateral. 
Afectación nerviosa: Los osteocondromas centrales y/o periféricos pueden producir síndromes de compresión nerviosa, pudiendo generar sintomatología de atrapamiento, siendo la del nervio peroneo causada por una lesión tibial, la más frecuente ${ }^{(1)}$. Más raramente se ha descrito la compresión del nervio ciático por lesiones del cuello femoral ${ }^{(4)}$. En este aspecto la RM es un excelente método de imagen, pudiendo demostrar el cambio de señal del nervio y la atrofia muscular con sustitución grasa de los músculos involucrados (Figura 11)(1,10). Por último, un dato interesante es que las lesiones centrales que producen compresión nerviosa en pacientes con exostosis hereditaria múltiple suelen ser solitarias ${ }^{(1)}$.

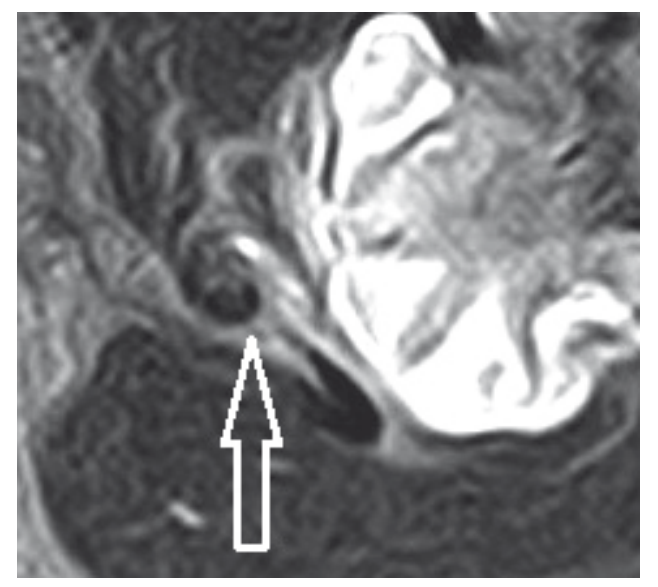

Figura 11. RM axial de cadera, secuencia T2 Stir, imagen ampliada: Osteocondroma del fémur izquierdo con capuchón cartilaginoso hiperintenso y desplazamiento sin cambios de señal del nervio ciático adyacente (flecha).

Formación de bursa: En general, se desarrolla en sitios donde existe fricción entre estructuras, es decir, entre la exostosis y una estructura adyacente ${ }^{(1)}$. Esto suele ocurrir alrededor de la escápula ${ }^{(11,28,29)}$, en la cadera y el hombro. Esta complicación se ve como una masa de partes blandas cercana a un osteocondroma, pudiendo contener áreas de mineralización condroide que pueden simular una malignización. La ecografía es muy útil para distinguir la colección bursal anecoica del tejido sólido hipoecoico de un capuchón de cartílago hialino. La bursa, a su vez, puede complicarse, pudiendo producirse infección, inflamación, o hemorragia ${ }^{(1)}$; la presencia de un bultoma debido a una bursa infectada reactiva puede sugerir clíncamente la malignización de una lesión ${ }^{(30)}$, En este aspecto, la RM es un excelente método de imagen que permite hacer el diagnóstico demostrando el realce periférico con contraste o la presencia de tabiques en el casos de sobreinfección (Figura $12 \mathrm{a}-\mathrm{c})^{(30)}$. Otro aspecto a tener en cuenta es la presencia de una condrometaplasia que derive en una osteocondromatosis secundaria a partir de la sinovial de la bursa ${ }^{(1,31)}$. Malignización:
La transformación maligna es la complicación más temida y se debe a un condrosarcoma que deriva del capuchón cartilaginoso de la lesión ${ }^{(1,7)}$. Las lesiones más susceptibles a transformación maligna son las de la pelvis, caderas y hombros. Los hallazgos que sugieren malignización consisten en crecimiento de una lesión previamente estable, márgenes irregulares, regiones radiolucentes en el interior, erosiones o destrucción del hueso adyacente y masa de partes blandas con calcificaciones irregulares. El criterio de imagen más importante a tener en cuenta es el grosor del capuchón cartilaginoso, habiendo diferencias entre autores en cuanto al valor patológico y normal de éste ${ }^{(1,32)}$. Debido a su alto contenido en agua, en la RM presenta alta intensidad de señal en T2 y baja en T1. Suele encontrarse adyacente a una zona de baja señal que representa el pericondrio ${ }^{(10)}$.

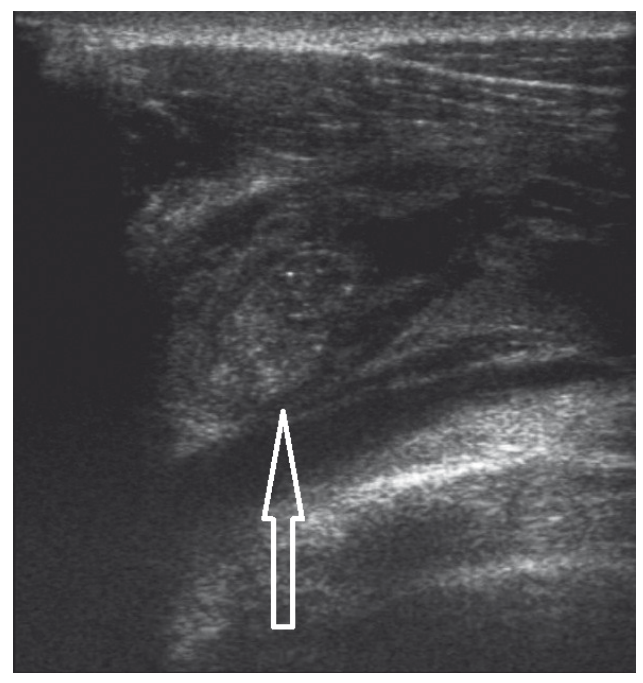

Figura 12a. Ecografía de tumoración dorsal: Imagen ovalada heterogénea de bordes bien definidos y de localización intramuscular subescapular, correspondiente a bursa infectada (flecha).

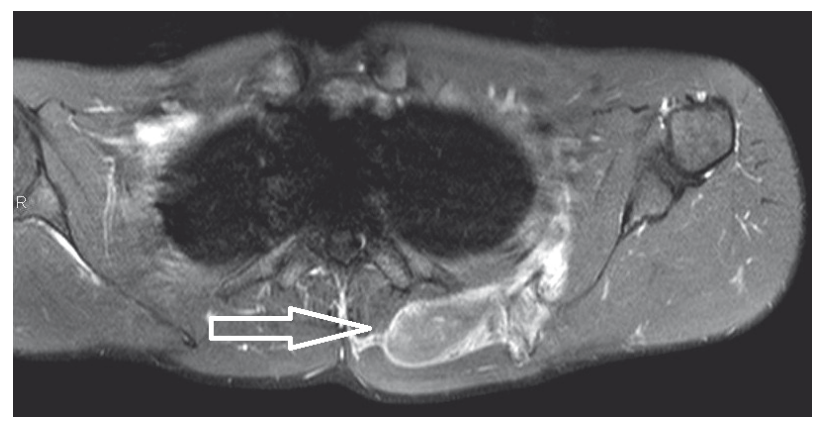

Figura 12b. RM Axial de pared torácica, secuencia T1 Spir con Gadolinio: Bursa entre osteocondroma escapular izquierdo (no visualizado) y pared torácica: imagen redondeada con captación periférica localizada a nivel d pared torácica posterior izquierda, que sugiere sobreinfección. 


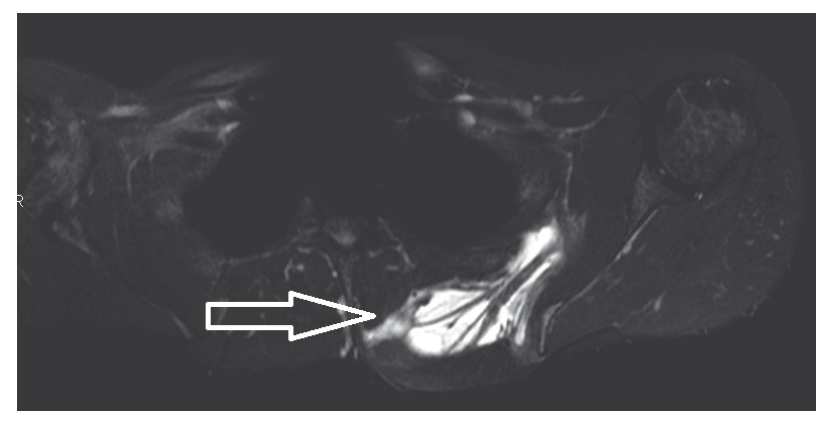

Figura 12c. RM axial de pared torácica, secuencia T2 Spir: Bursa entre osteocondroma escapular izquierdo (no visualizado) y pared torácica: Imagen hiperintensa e irregular que corresponde a bursa (flecha) con tabiques hipointensos en su interior, que sugieren sobreinfección.

Variantes: Displasia epifisaria hemimelica (enfermedad de Trevor). Esta entidad consiste en un crecimiento osteocartilaginoso de una o varias epífisis en su lado medial o lateral ${ }^{(1,33,34)}$. Afecta mayormente a los huesos largos del miembro inferior y huesos del tarso, en especial la rodilla, el tobillo y el astrágalo ${ }^{(8,35)}$. Existen casos raros reportados a nivel de la cadera, patela, la columna ${ }^{(36-38)}$ y en la mano ${ }^{(39)}$. Se describen tres formas, una localizada, una clásica, afectando más de un área en una sola extremidad, y una forma generalizada, considerándose rara la afectación bilateral| ${ }^{(1,40)}$. La primera suele afectar al tobillo; la segunda afecta a epífisis, más frecuentemente alrededor de la rodilla y tobillo; y la última, a toda la extremidad inferior ${ }^{(1,34)}$ (Figuras 13 y 14 a-c). Los hallazgos radiológicos en pacientes pediátricos muestran los centros de osificación afectados prematuramente excéntricos, lobulados y aumentados de tamaño. Además, pueden visualizarse calcificaciones. Posteriormente, se visualiza la típica exostosis epifisaria con continuidad cortical y medular. La TC y la RM, permiten visualizar estos aspectos, además del detalle del componente cartilaginoso. Se pueden ver, además, áreas con señal baja en T1 y T2, que representan zonas de calcificación u osificación ${ }^{(1,41)}$. En el diagnóstico diferencial se debe tener en cuenta a los osteocondromas, aunque las lesiones del Trevor ocurren en pacientes pediátricos y adolescentes, y se dan en las epífisis. El osteocondroma, sin embargo, ocurre entre los 10 y 30 años y se origina en las metáfisis de los huesos largos ${ }^{(42)}$.

Otras Variantes: Otras variantes más raras comprenden la exostosis de Turret, que consiste en una masa extracortical dependiente de las falanges proximales o medias de la mano. Lesiones similares han sido descritas a nivel de inserciones tendinosas y ligamentosas, denominadas exóstosis de tracción. Lesiones similares han sido descritas en pies y manos, la periostitis reactiva florida y la proliferación osteocondromatosa paraostal o lesión de Nora(1). Esta última afecta a pacientes entre la tercera y cuarta décadas de vida, sin preferencia de sexo(9). Consiste en un crecimiento exofítico desde una superficie ósea cortical y está compuesto de cartílago, hueso y tejido fibroso, afectando principalmente a las falanges proximales y medias y a los metacarpianos o metatarsianos ${ }^{(43)}$ (Figura 15 $a, b)$. Las radiografías revelan, en un estadio más avanzado, una excrecencia ósea, con una base de separación entre la lesión y el hueso, debida a la existencia de tejido cartilaginoso interpuesto entre ambos, aunque también puede verse continuidad con la lesión en algunos casos(1). El TC además de demostrar estos hallazgos, permite evaluar la existencia o ausencia de continuidad con la cortical adyacente y la existencia de afectación de partes blandas. El diagnóstico final lo brinda la anatomía patológica, ya que estas lesiones pueden ser confundidas con un osteosarcoma paraostal y/o un osteocondroma convencional. El primero, raramente afecta a huesos tan pequeños, y el segundo, además del análisis histopatológico, posee otras características como la continuidad cortical y medular. Por último, esta lesión puede ser confundida con un proceso maligno, debido a la alta tasa de recurrencia que posee ${ }^{(1,43)}$.

\section{Conclusión}

El osteocondroma representa el tumor óseo más común, y posee unas características radiológicas típicas, principalmente la continuidad cortical y medular.

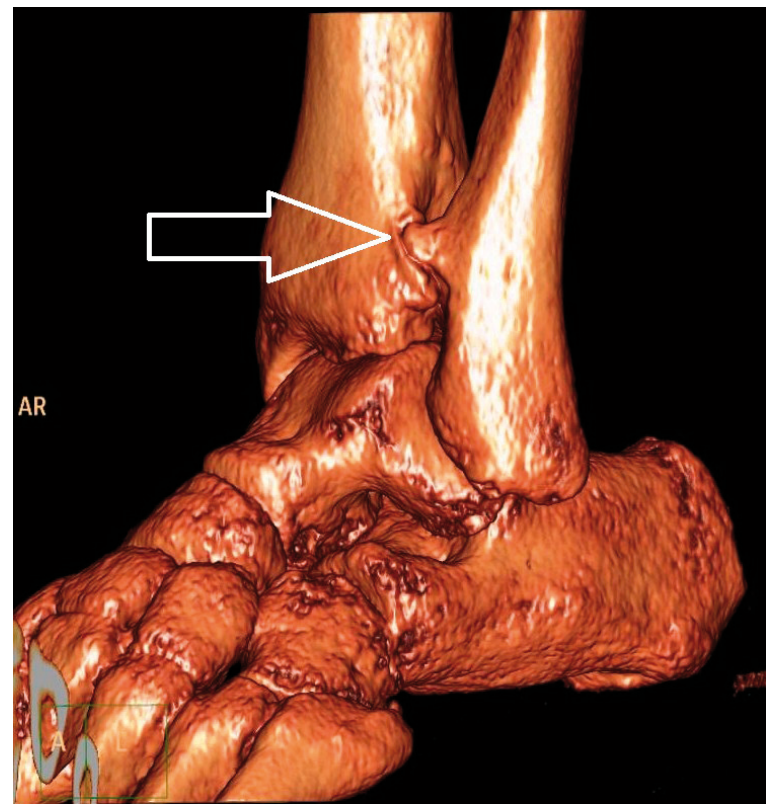

Figura 13. TC de tobillo izquierdo, reconstrucción $3 D$, visión antero externa: Enfermedad de Trevor: lesión ósea dependiente de articulación tibioastragalina distal (flecha), que produce remodelamiento de mortaja tibioperonea. 


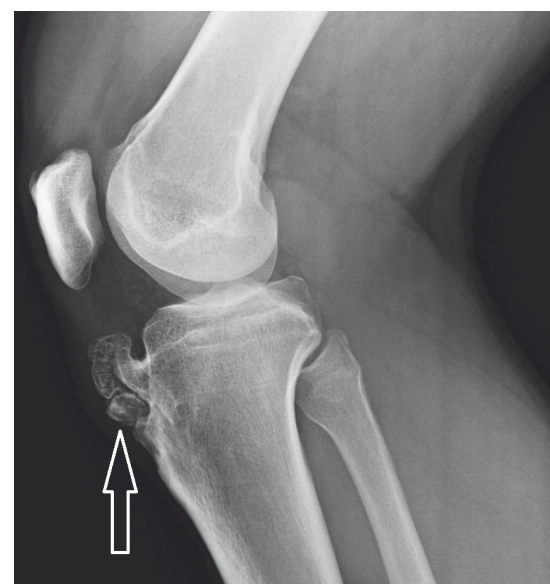

Figura 14a. $R X$ lateral de rodilla: Enfermedad de Trevor: lesión ósea exofítica dependiente de tuberosidad tibial anterior (flecha), con aspecto de "cuerno" correspondiente a osteocondroma epifisario.

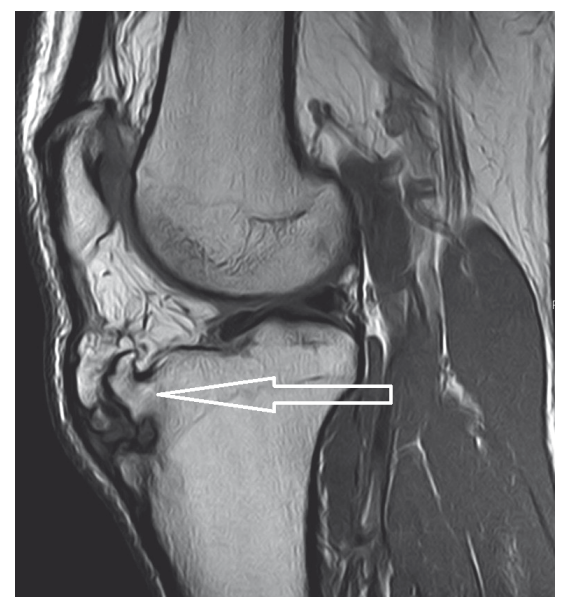

Figura 14b. RM rodilla, secuencia sagital T1: Enfermedad de Trevor: lesión ósea exofítica con continuidad cortico medular dependiente de tuberosidad tibial anterior (flecha), con aspecto de "cuerno", correspondiente a osteocondroma epifisario.

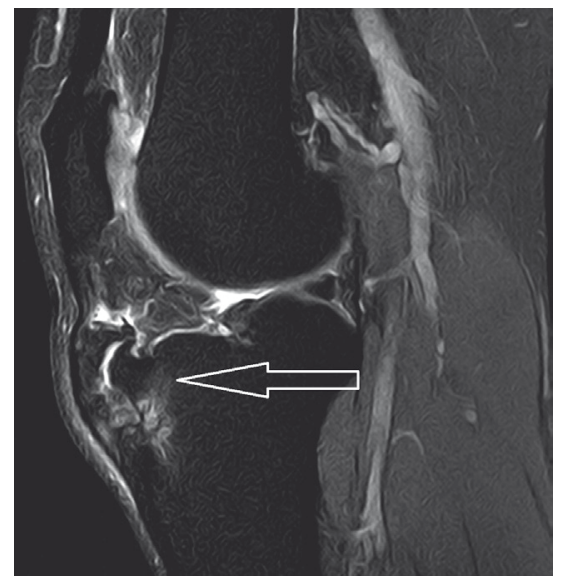

Figura 14c. RM rodilla, secuencia sagital DP Spir: Enfermedad de Trevor: lesión ósea exofítica con continuidad cortico medular dependiente de tuberosidad tibial anterior (flecha) compatible con Osteocondroma epifisario, observándose área hiperintensa en su base correspondiente a edema.

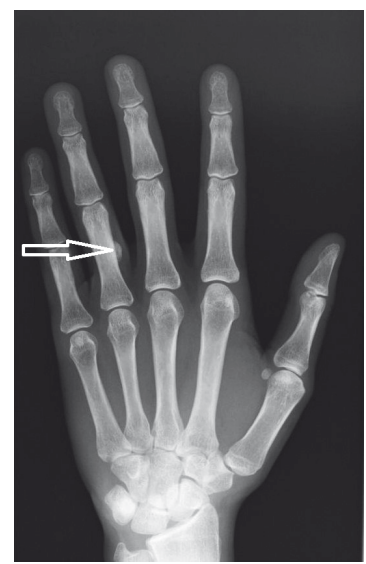

Figura 15a. $R X A P$ de mano izquierda: Lesión de Nora: tumoración ósea cortical de aspecto benigno, dependiente de vertiente radial de diáfisis de falange proximal de 4to. Dedo

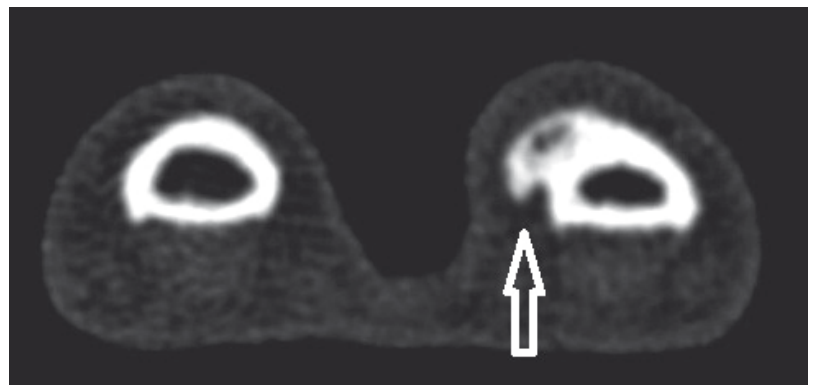

Figura 15c. TC axial de mano, 2 y 3er dedos: Lesión de Nora: tumoración ósea cortical dependiente de falange de 4to. Dedo y de aspecto benigno.

Ante casos dudosos y para el estudio de variantes y/o complicaciones, pueden necesitarse otras pruebas de imagen con el TC o en especial la RM, que permiten llegar al diagnóstico en la mayoría de los casos. El conocimiento del espectro de hallazgos en esta lesión permite que el radiólogo pueda realizar un correcto diagnóstico y que ayude en dirigir el manejo del paciente hacia una terapéutica correcta.

\section{Bibliografía}

1. Murphey MD, Choi JJ, Kransdorf MJ, Flemming DJ, Gannon FH. Imaging of osteochondroma: variants and complications with radiologic-pathologic correlation. Radiographics 2000 Sep-Oct; 20(5): 1407-1434.

2. Bovée JV. Múltiple osteochondromas. Orphanet J Rare Dis 2008 Feb 13; 3 : 3.

3. Malhotra K, Nunn T, Chandramohan M, Shanker J. Metatarsal stress fractures secondary to soft-tissue osteochondroma in the foot: case report and literature review. Foot Ankle Surg $2011 \mathrm{Dec}$; 17(4): e51-54.

4. Yu K, Meehan JP, Fritz A, Jamali AA. Osteochondroma of the femoral neck: a rare cause of sciatic nerve compression. Orthopedics 2010 Aug 11; 33(8. doi: 10.3928/0147744720100625-26.

5. Gruber-Szydło K, Poreba R, Belowska-Bien K, Derkacz A, Badowski R, Andrzejak R. Popliteal artery thrombosis secondary to a tibial osteochondroma. Vasa 2011 May; 40(3): 251-5.

6. Mahmoodi SM, Bahirwani RK, Abdull-Gaffar BA, Habib IF. Intrabursal vein abrasion and thrombosis. An unusual complication of femoral osteochondroma. Saudi Med J 
2009 Dec; 30(12): 1604-1606.

7. Martin C, Munk PL, O'Connell JX, Lee MJ, Masri B, Wambeek N. Malignant degeneration of an osteochondroma with unusual intra-bursal invasion. Skeletal Radiol 1999 Sep; 28(9): 540-543.

8. Azzoni R. Dysplasia epiphysealis hemimelica of the talus.J Orthop Traumatol. 2009 Mar; 10(1): 43-46.

9. Adler D, Aigner T, et al. Nora's lesion. Discussion of a rare bone proliferation. Orthopade 2010 Nov; 39(11): 1065-1070.

10. Kitsoulis P, Galani V, Stefanaki K, Paraskevas G, et al. Osteochondromas: review of the clinical, radiological and pathological features. In Vivo 2008 sep-oct; 22(5): 633-646.

11. Pérez D, Ramón Cano J, Caballero J, López L. Minimallyinvasive resection of a scapular osteochondroma. Interact Cardiovasc Thorac Surg 2011 nov; 13(5): 468-470.

12. Mohsen MS, Moosa NK, Kumar P. Osteochondroma of the scapula associated with winging and large bursa formation. Med Princ Pract 2006; 15(5): 387-390.

13. Katayama T, Ono H, Furuta K. Osteochondroma of the lunate with extensor tendons rupture of the index finger: a case report. Hand Surg. 2011; 16(2): 181-184.

14. Frost NL, Parada SA, Manoso MW, Arrington E, Benfanti P. Scapular osteochondromas treated with surgical excision. Orthopedics 2010 nov 2; 33(11): 804. doi: 10.3928/01477447-20100924-09.

15. Rodallec MH, Feydy A, Larousserie F, Anract P, Campagna $R$, Babinet $A$, et al. Diagnostic imaging of solitary tumors of the spine: what to do and say. Radiographics 2008 Jul-Aug; 28(4): 1019-1041.

16. Kumar A, Rastogi S, Modi M, Nijhawan S. Osteochondroma of the mandibular condyle. Indian J Dent Res 2011 Jul-Aug; 22(4): 616. Doi:10.4103/0970-9290.90330.

17. Utumi ER, Pedron IG, Perrella A, Zambon CE, Ceccheti MM, Cavalcanti MG. Osteochondroma of the temporomandibular joint: a case report. Braz Dent J 2010; 21(3): 253-258.

18. De Maio F, Bisicchia S, Potenza V, Caterini R, Farsetti P. Giant intra-articular extrasynovial osteochondroma of the knee: a report of two cases. Open Orthop J 2011; 5: 368-371.

19. Kose O, Ertas A, Celiktas M, Kisin B. Fracture of an osteochondroma treated successfully with total excision: two case reports. Cases J 2009 aug 7; 2: 8062.

20. Scotti C, Marone EM, Brasca LE, Peretti GM, Chiesa $R$, Del Maschio A, et. al Pseudoaneurysm overlying an osteochondroma: a noteworthy complication. J Orthop Traumatol 2010 Dec; 11(4): 251-255.

21. Toumi S, Ghnaya H, Essid A, Braham A, Jerbi S, MradDaly K, et. al. Hereditary multiple exostosis revealed by deep vein and arterial popliteal thrombosis. Rev Med Interne 2010 apr; 31(4): e7-10.

22. Mehta M, White LM, Knapp T, Kandel RA, Wunder JS, Bell RS. MR imaging of symptomatic osteochondromas with pathological correlation. Skeletal Radiol 1998 aug; 27(8): 427-433.

23. Lee KC, Davies AM, Cassar-Pullicino VN. Imaging the complications of osteochondromas. Clin Radiol 2002 jan; 57(1):18-28.

24. Oudyi M, David M, Blondel B, Bosdure E, Gorincour G, Launay $F$, et. al. Hemothorax and hereditary multiple exostosis in a 9-year-old boy. Arch Pediatr 2011 feb; 18(2): $170-175$.
25. Rupprecht M, Mladenov K, Stücker R. Posttraumatic popliteal pseudoaneurysm caused by a femoral osteochondroma. J Pediatr Orthop B 2010 jul; 19(4): 341-343.

26. Gruber-Szydło K, Poreba R, Belowska-Bien K, Derkacz A, Badowski R, Andrzejak R, et al. Popliteal artery thrombosis secondary to a tibial osteochondroma. Vasa 2011 may; 40(3): 251-255

27. O'Brien PJ, Ramasunder S, Cox MW. Venous thoracic outlet syndrome secondary to first rib osteochondroma in a pediatric patient. J Vasc Surg 2011 mar; 53(3): 811-813.

28. Yoo WH, Kim JR, Jang KY, Lee SY, Park JH. Rapidly developed huge bursitis associated with scapular osteochondroma of the multiple exostosis: a case report. Rheumatol Int 2009 jan; 29(3): 317-319.

29. Shackcloth MJ, Page RD. Scapular osteochondroma with reactive bursitis presenting as a chest wall tumour. Eur J Cardiothorac Surg. 2000 oct; 18(4): 495-496.

30. Koplas M, Winalski C, Sundaram M. What's your diagnosis? Osteochondroma with large bursa clinically mimicking malignant transformation. Orthopedics $2007 \mathrm{Jul} ; 30$ (7): 505, 581-583.

31. Errani C, Jutte PC, De Paolis M, Bacchini P, Mercuri M. Secondary synovial chondromatosis in a bursa overlying an osteochondroma mimicking a peripheral chondrosarcoma - a case report. Acta Orthop 2007 oct; 78(5): 701-704.

32. Passanise AM, Mehlman CT, Wall EJ, Dieterle JP. Radiographic evidence of regression of a solitary osteochondroma: a report of 4 cases and a literature review. J Pediatr Orthop 2011 apr-may; 31(3): 312-316.

33. Volders D, Vandevenne JE, Van de Casseye W. Trevor's disease and whole-body MRI. Eur J Radiol 2011 sep; 79(3): 363-364.

34. Masquijo JJ, Willis B. Dysplasia epiphysealis hemimelica (Trevor's disease). Arch Argent Pediatr 2010 feb; 108(1): e20-23.

35. Gokkus K, Aydin AT, Uyan A, Cengiz M. Dysplasia epiphysealis hemimelica of the ankle joint: a case report. Orthop Surg (Hong Kong) 2011 aug; 19(2): 254-256.

36. Araujo CR Jr, Montandon S, Montandon C, Teixeira $\mathrm{KI}$, Moraes FB, Moreira MA.Best cases from the AFIP: dysplasia epiphysealis hemimelica of the patella. Radiographics 2006 Mar-Apr; 26(2): 581-586.

37. Wenger DR, Adamczyk MJ. Evaluation, imaging, histology and operative treatment for dysplasia epiphysealis hemimelica (Trevor disease) of the acetabulum: a case report and review. lowa Orthop J 2005; 25: 60-65.

38. Perl M, Brenner RE, Lippacher S, Nelitz M. Dysplasia epiphysealis hemimelica: a case report with novel pathophysiologic aspects. Clin Orthop Relat Res 2009 sep; 467(9): 2472-2478.

39. Gölles A, Stolz P, Freyschmidt J, Schmitt R. Trevor's disease (dysplasia epiphysealis hemimelica) located at the hand: case report and review of the literature. Eur $\mathrm{J}$ Radiol 2011 feb; 77(2): 245-248.

40. Wiart E, Budzik JF, Fron D, Herbaux B, Boutry N. Bilateral dysplasia epiphysealis hemimelica of the talus associated with a lower leg intramuscular cartilaginous mass. Pediatr Radiol 2012 apr; 42(4): 503-507.

41. Bahk WJ, Lee HY, Kang YK, Park JM, Chun KA, Chung YG. Dysplasia epiphysealis hemimelica: radiographic and magnetic resonance imaging features and clinical outcome of complete and incomplete resection. Skeletal Radiol 2010 jan; 39(1): 85-90. 